\title{
Nonlinear vibrations of thin hyperelastic plates
}

\author{
Ivan D. Breslavsky, Marco Amabili*, Mathias Legrand \\ Department of Mechanical Engineering, McGill University, 817 Sherbrooke Street West, Montréal, Québec, Canada H3A 0C3
}

\section{A R T I C L E I N F O}

\section{Article history:}

Received 6 January 2014

Received in revised form

21 March 2014

Accepted 14 April 2014

Handling Editor: M.P. Cartmell

Available online 20 May 2014

\begin{abstract}
A B S T R A C T S
Static deflection as well as free and forced nonlinear vibration of thin square plates made of hyperelastic materials are investigated. Two types of materials, namely rubber and soft biological tissues, are considered. The involved physical (material) nonlinearities are described through Neo-Hookean, Mooney-Rivlin, and Ogden hyperelastic laws; geometrical nonlinearities are modeled by the Novozhilov nonlinear shell theory. Dynamic local models are first built in the vicinity of a static configuration of interest that has been previously calculated. This gives rise to the approximation of the plate's behavior in the form of a system of ordinary differential equations with quadratic and cubic nonlinear terms in displacement. Numerical results are compared and validated in the static case via a commercial FE software package: they are found to be accurate for deflections reaching 100 times the thickness of the plate. The frequency shift between low- and large-amplitude vibrations weakens with an increased initial deflection.
\end{abstract}

(c) 2014 Elsevier Ltd. All rights reserved.

\section{Introduction}

Thin-walled structures made of hyperelastic materials, such as rubbers and biomaterials, are common in mechanical and biomechanical engineering applications. Such structures are frequently subjected to significant static and dynamic loadings potentially yielding large deflections and deformations. Associated previous works reported in the literature employ, in a vast majority, a simplifying assumption that considers as a priori known the shape of the deformed structure; for instance, a cylindrical shell remains cylindrical after deformation. A general review on these works is available in [1]. The present investigation discards this limitation.

One possible way to account for complicated deformed shapes is to use the finite element (FE) approach. For instance, Einstein et al. [2] developed a finite element strategy to analyze the dynamic behavior of hyperelastic and viscoelastic membranes with amplitudes of the order of their thickness. With the proposed method, the dynamic response of a hemisphere made of Mooney-Rivlin material under impact pressure load is studied. A relatively similar problem concerning the dynamic inflation of a Mooney-Rivlin spherical membrane is explored in [3] also through the FE method.

Another technique lies in the approximation of the deformed configuration as a truncated series of continuous functions satisfying the essential (geometric) boundary conditions. This approach is implemented for circular membranes in $[4,5]$. The sensitivity of the respective vibratory behavior to the pre-stretch configuration of the membranes is carried out in these

\footnotetext{
* Correspondence to: Department of Mechanical Engineering, McGill University, 817 Sherbrooke Street West, Montreal, Canada H3A 2 K6.

Tel.: +1514 3983068 .

E-mail address: marco.amabili@mcgill.ca (M. Amabili).

URL: http://people.mcgill.ca/marco.amabili/ (M. Amabili).
} 
scientific contributions. The incompressible hyperelastic material is either described by a Neo-Hookean model [5] or by Mooney-Rivlin, Yeoh, Ogden, and Arruda-Boyce models in [4]. In this latter work, the frequency-amplitude relationships for various hyperelastic models are found to be analogous. The authors come to the conclusion that the higher the stretch, the closer the nonlinear forced response to its linear counterpart.

In [1], the static deflection and vibration around a deformed configuration of a rectangular Neo-Hookean plate are investigated accounting for both geometrical (the nonlinearity of strain-displacements relations) and physical nonlinearities (the material nonlinearity of stress-strain relations). Is proposed a method which systematically builds approximate local models (LMM) in the form of polynomial expansions of the non-polynomial strain energy densities: this greatly simplifies the description of the plate's dynamic behavior. The present paper extends this investigation to various hyperelastic laws by using a more sophisticated nonlinear plate theory. The two targeted types of material (rubber and biomaterial) are described through (i) Neo-Hookean, (ii) Mooney-Rivlin, and (iii) Ogden hyperelastic laws as well as (iv) a physically linear material law. The comparison of static force-deflection curves and frequency responses obtained with these models is carried out and the contribution of in-plane nonlinearities in the sought displacement is estimated. The numerical solutions are verified through direct comparison with results from a commercial FE software package.

\section{Kinematics and energy equations}

The geometrical nonlinearity in the kinematics of the investigated flexible plate is described with the help of Novozhilov nonlinear plate theory [6], which stands as a limit case of Novozhilov nonlinear shell theory. It is governed by the following strain-displacement relationships:

$$
\begin{gathered}
\varepsilon_{1}=u_{x}+\frac{1}{2}\left(w_{x}^{2}+\left[u_{x}^{2}+v_{x}^{2}\right]\right)-z w_{x x} ; \\
\varepsilon_{2}=v_{y}+\frac{1}{2}\left(w_{y}^{2}+\left[u_{y}^{2}+v_{y}^{2}\right]\right)-z w_{y y} ; \\
\varepsilon_{12}=u_{y}+v_{x}+w_{x} w_{y}+\left[u_{x} u_{y}+v_{x} v_{y}\right]-2 z w_{x y},
\end{gathered}
$$

where $\varepsilon_{1}, \varepsilon_{2}$, and $\varepsilon_{12}$ are the components of the Green-Lagrange strain tensor for thin plates. Novozhilov nonlinear plate theory, commonly recognized as the finest classical plate theory, reduces to the well-known von Kármán nonlinear plate theory [6] when the in-plane terms in square brackets in (1) are neglected. Although it neglects shear deformations and rotary inertia, it is accurate for thin plates. Also, it is known that in-plane nonlinearities play a major role in large deflections, and they are accordingly retained in the present work to improve accuracy.

Lagrange equations are utilized to derive the dynamics of the plate, that is

$$
\frac{d}{\mathrm{~d} t}\left(\frac{\partial L}{\partial \dot{q}_{n}}\right)+\frac{\partial L}{\partial q_{n}}=Q_{n}, \quad n=1, \ldots, N
$$

where $L=T-\Pi$ is Lagrange's functional, $T$ is the kinetic energy of the plate, $\Pi$ is the potential elastic deformation energy, and $Q_{n}$ are the generalized forces. The potential and kinetic energies are expressed as follows [6]:

$$
\begin{gathered}
\Pi=\iiint_{V} W \mathrm{~d} V, \\
T=\frac{\rho h}{2} \iint_{S}\left(\dot{u}^{2}+\dot{v}^{2}+\dot{w}^{2}\right) \mathrm{d} S,
\end{gathered}
$$

where $W$ is the strain energy density (SED), $V$ is the volume of the plate, $S$ is the surface of the middle plane of the plate, $\rho$ is the mass-density of the plate material, $h$ is the thickness of the plate and $u, v, w$ are the displacements along the axes of the rectangular coordinate system $x, y, z$, respectively. The dot stands for differentiation with respect to time.

The displacements are expanded into corresponding truncated series involving the appropriate generalized coordinates $q_{n}:$

$$
\begin{gathered}
w(x, y, t)=\sum_{i=1}^{N_{w}} q_{i}(t) W_{i}(x, y), \\
u(x, y, t)=\sum_{i=1}^{N_{U}} q_{i+N_{w}}(t) U_{i}(x, y), \\
v(x, y, t)=\sum_{i=1}^{N_{V}} q_{i+N_{U}+N_{w}}(t) V_{i}(x, y)
\end{gathered}
$$

In Eq. (5), quantities $W_{i}, U_{i}, V_{i}$ are the admissible functions that satisfy the homogeneous boundary conditions (i.e. the geometric constraints) of the problem. The linear modes of vibration, which form a complete set properly capturing the dynamics of a structure, are eligible admissible functions and are selected in the present work. The total number of degrees of freedom is

$$
N=N_{W}+N_{U}+N_{V} .
$$


Table 1

SEDs for various incompressible hyperelastic laws.

\begin{tabular}{ll}
\hline Material law & Strain energy density \\
\hline Neo-Hookean $[7,8]$ & $W=\frac{E}{6}\left(I_{1}-3\right)$ \\
Mooney-Rivlin $[7,8]$ & $W=\frac{\mu_{1}}{2}\left(I_{1}-3\right)+\frac{\mu_{2}}{2}\left(I_{2}-3\right)$ \\
Ogden [7] & $W=\sum_{i=1}^{N_{T}} \frac{\mu_{i}}{\alpha_{i}}\left(\lambda_{1}^{\alpha_{i}}+\lambda_{2}^{\alpha_{i}}+\lambda_{3}^{\alpha_{i}}-3\right)$ \\
Linear material $[1,6]$ & $W=\frac{E}{6}\left(4\left(\varepsilon_{1}^{2}+\varepsilon_{2}^{2}+\varepsilon_{1} \varepsilon_{2}\right)+\varepsilon_{12}^{2}\right)$. \\
\hline
\end{tabular}

\section{Hyperelastic relations}

\subsection{Strain energy density}

Usually, nonlinear elasticity of rubbers and soft biomaterials is described by hyperelastic laws and in most cases, such materials are assumed to be incompressible [7,8]. Three hyperelastic laws together with their associated SEDs, listed in Table 1, are considered: Neo-Hookean, Mooney-Rivlin, and Ogden laws.

In Table 1, the following notation is used: $I_{1}$ is the first invariant of the right Cauchy-Green deformation tensor C; $E$ is Young's modulus of the plate's material; $I_{2}$ stands for the second invariant of the right Cauchy-Green deformation tensor; $\lambda_{1}, \lambda_{2}, \lambda_{3}$ are the principal stretches of the plate; $\mu_{i}, \alpha_{i}$ denote the material parameters.

Since SED (9) is polynomial in strains, spatial and temporal components of the solution can be uncoupled and the Lagrange equations reduce to simple ordinary differential equations with quadratic and cubic nonlinearities [6]

$$
\begin{aligned}
& \ddot{q}_{n}+2 \zeta_{n} \Omega_{n} \dot{q}_{n}+\sum_{i=1}^{N} k_{n i} q_{i}+\sum_{i, j=1}^{N} k_{n i j} q_{i} q_{j}+\sum_{i, j, l=1}^{N} k_{n i j l} q_{i} q_{j} q_{l}=Q_{n}, \\
& n=1, \ldots, N
\end{aligned}
$$

where $\Omega_{n}$ is the natural frequency of mode $n$ and $\zeta_{n}$ is the corresponding damping ratio; $k_{n i}, k_{n i j}, k_{n i j l}$ are known coefficients that result from integration in space.

\subsection{Cauchy-Green strain tensor invariants and principal stretches}

In order to derive the expressions of the invariants of the right Cauchy-Green deformation tensor $\mathbf{C}$ in terms of displacements, the Green-Lagrange strain tensor

$$
\mathbf{E}=\frac{1}{2}\left(\begin{array}{lll}
2 \varepsilon_{1} & \varepsilon_{12} & 0 \\
\varepsilon_{12} & 2 \varepsilon_{2} & 0 \\
0 & 0 & 2 \varepsilon_{3}
\end{array}\right)
$$

is used, where the expressions of $\varepsilon_{1}, \varepsilon_{2}, \varepsilon_{12}$ (but not $\varepsilon_{3}$ ) are given in (1). The right Cauchy-Green deformation tensor $\mathbf{C}$ is then defined as [8]

$$
\mathbf{C}=2 \mathbf{E}+\mathbf{I}=\left(\begin{array}{lll}
2 \varepsilon_{1}+1 & \varepsilon_{12} & 0 \\
\varepsilon_{12} & 2 \varepsilon_{2}+1 & 0 \\
0 & 0 & 2 \varepsilon_{3}+1
\end{array}\right)
$$

and its three invariants are

$$
\begin{gathered}
I_{1}=\operatorname{Tr}(\mathbf{C})=2\left(\varepsilon_{1}+\varepsilon_{2}+\varepsilon_{3}\right)+3 ; \\
I_{2}=\frac{1}{2}\left(\operatorname{Tr}(\mathbf{C})^{2}-\operatorname{Tr}\left(\mathbf{C}^{2}\right)\right)= \\
4\left(\varepsilon_{1}+\varepsilon_{2}+\varepsilon_{3}+\varepsilon_{1} \varepsilon_{2}+\varepsilon_{1} \varepsilon_{3}+\varepsilon_{2} \varepsilon_{3}\right)-\varepsilon_{12}^{2}+3 ; \\
J^{2}=|\mathbf{C}|=\left(2 \varepsilon_{3}+1\right)\left(\left(2 \varepsilon_{1}+1\right)\left(2 \varepsilon_{2}+1\right)-\varepsilon_{12}^{2}\right) .
\end{gathered}
$$


The third invariant $J$ is used to reflect the incompressibility condition through $J=1$ [7] and the principal stretches are the square roots of the eigenvalues of $\mathbf{C}[8]$

$$
\begin{gathered}
\lambda_{1,2}=\sqrt{1+\varepsilon_{1}+\varepsilon_{2} \pm \sqrt{\left(\varepsilon_{1}-\varepsilon_{2}\right)^{2}+\varepsilon_{12}^{2}}} \\
\lambda_{3}=\sqrt{1+2 \varepsilon_{3}}
\end{gathered}
$$

\subsection{Transverse normal strain}

The transverse normal strain $\varepsilon_{3}$ expressed in terms of $u, v$, and $w$ shall be inserted into expressions (13) to (17) and the incompressibility condition (15) is enforced to retrieve the expression of $\varepsilon_{3}$

$$
\varepsilon_{3}=\frac{1}{2\left(\left(2 \varepsilon_{1}+1\right)\left(2 \varepsilon_{2}+1\right)-\varepsilon_{12}^{2}\right)}-\frac{1}{2} .
$$

Expression (18) is then introduced in (13)-(15) and (17) as well as in SEDs (6)-(8).

\section{Local expansion of the strain energy density}

Expressions (6)-(8) together with $\varepsilon_{3}$ from (18) are not polynomials in strains, which essentially complicates the investigation of the plate's behavior. The analysis is thus simplified by introducing a transformation of SEDs (6)-(8) in order to derive approximate governing equations in the form of ordinary differential equations with nonlinearities of order not higher than three. The corresponding local model is reliable only in the vicinity of a configuration of interest around which the SED is expanded into a series in the generalized coordinates truncated at order 4 . To reach highly deformed configurations, successive local models have to be constructed. Further details on the local models method (LMM) can be found in [1].

\section{Exact low-dimensional models with both material and geometric nonlinearities}

A distinct additional approach is implemented to measure the accuracy of the LMM. It involves the numerical solution of Eq. (2) in its static version, that is

$$
\iiint_{V} \frac{\partial W}{\partial q_{n}} \mathrm{~d} V=Q_{n}, \quad n=1, \ldots, N
$$

with SEDs (6)-(8) and expression (18) previously substituted into it. Since the latter is a smooth function in the generalized coordinates, integration and differentiation operators commute, that is

$$
\frac{\partial}{\partial q_{n}} \iiint_{V} W \mathrm{~d} V=\iiint V \frac{\partial W}{\partial q_{n}} \mathrm{~d} V
$$

System (19) of nonlinear algebraic equations can be solved numerically through the Newton-Raphson iterative technique; this approach is named the "exact solution" hereinafter.

\section{Numerical example: static and dynamic bending of a rubber plate}

\subsection{Hyperelastic models parameter identification}

Experimental data for 8 percent sulfur rubber obtained by Treloar [9] for uniaxial and equibiaxial tensions, as well as for pure shear, are exploited. This common rubber is chosen because experimental data for uniaxial and multiaxial loads is available in the literature: a good approximation for the strain energy density is then possible for this material. Corresponding details on the approximation of the experimental data are provided in Appendix A.

From the given experimental data, Young's modulus is $E=1,247,060.2 \mathrm{~Pa}$, which is in almost perfect agreement with Ogden's one, $E=1,242,992.9$ Pa [7]. Other relevant parameters are listed in Table 2.

Figs. 1-3 display the stress-strain relationships for the three hyperelastic laws together with the experimental points. The nominal stress $S_{1}$ versus engineering strain $\varepsilon$ is shown - see Appendix A for details. It is worthy to note that Neo-Hookean and Mooney-Rivlin laws are very close and both laws satisfactorily approximate the rubber behavior at

Table 2

Hyperelastic law parameters for rubber.

\begin{tabular}{ll}
\hline Neo-Hookean & $E=1,247,060.2 \mathrm{~Pa}$ \\
Mooney-Rivlin & $\mu_{1}=416,185.5 \mathrm{~Pa}, \mu_{2}=-498.8 \mathrm{~Pa}$ \\
Ogden & $\mu_{1}=161.2 \mathrm{~Pa}, \mu_{2}=-1831 \mathrm{~Pa}, \mu_{3}=781,111 \mathrm{~Pa}$, \\
& $\alpha_{1}=7.295, \alpha_{2}=-2.729, \alpha_{3}=1.056$
\end{tabular}




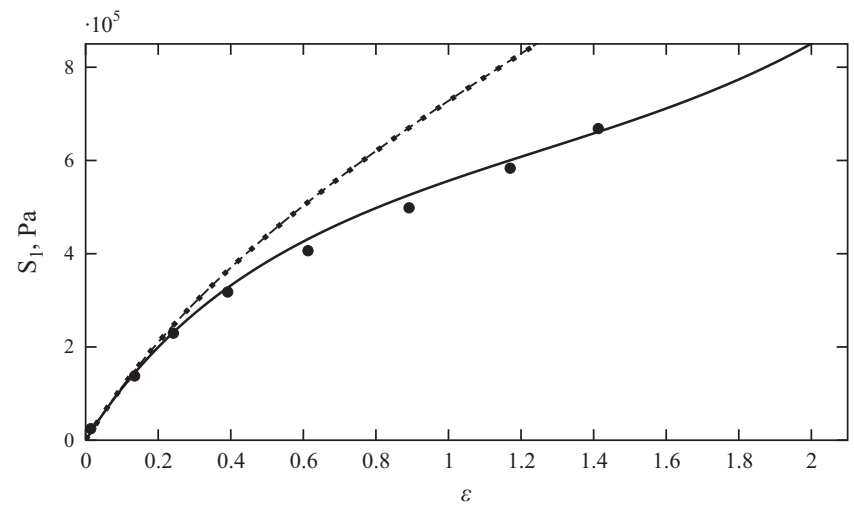

Fig. 1. Strain-stress curves and experimental points for rubber uniaxial tension. Dotted line: Neo-Hookean law; dashed line: Mooney-Rivlin law; continuous line: Ogden law.

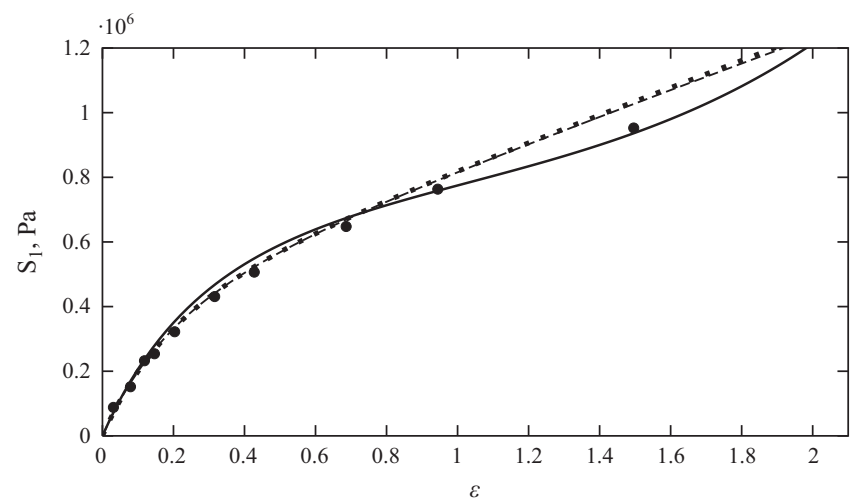

Fig. 2. Strain-stress curves and experimental points for rubber equibiaxial tension. Dotted line: Neo-Hookean law; dashed line: Mooney-Rivlin law; continuous line: Ogden law.

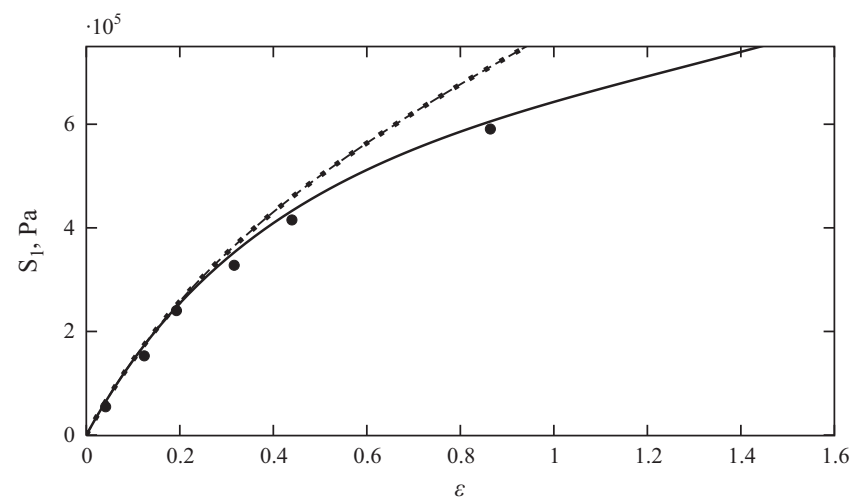

Fig. 3. Stress-strain curves and experimental points for pure shear of rubber. Dotted line, Neo-Hookean law; dashed line, Mooney-Rivlin law; continuous line, Ogden law.

strains not higher than 30 percent (that is, 0.3 in Figs. 1-3). However, the approximation of the rubber behavior offered by Ogden law is much more accurate on the full range of experimental strains.

\subsection{Problem description}

A simply supported square rubber plate illustrated in Fig. 4 is considered. It is defined on the following domain:

$$
V=\left\{x \in[0, a] ; y \in[0, b] ; z \in\left[\frac{-h}{2}, \frac{h}{2}\right]\right\},
$$




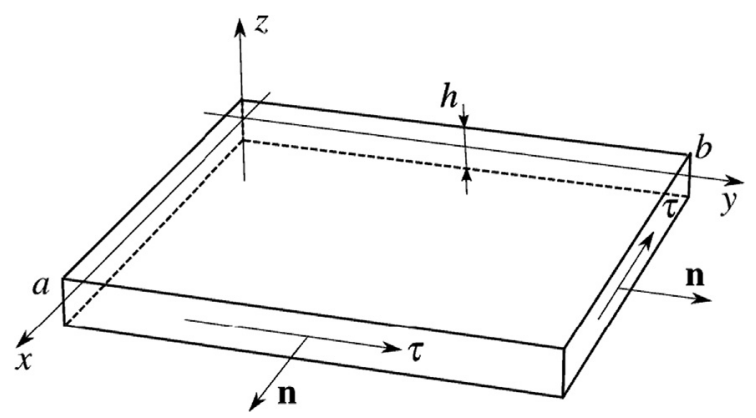

Fig. 4. Rectangular plate and coordinate system.

with $a=0.1 \mathrm{~m}, b=0.1 \mathrm{~m}, h=0.0005 \mathrm{~m}$. The material characteristics are listed in Table 2 and the rubber mass density is $\rho=1100 \mathrm{~kg} / \mathrm{m}^{3}$.

The plate is simply supported with immovable edges yielding the following boundary conditions [6]:

$$
\left.w\right|_{\partial S}=\left.M\right|_{\partial S}=\left.u\right|_{\partial S}=\left.v\right|_{\partial S}=0
$$

where $\partial S$ denotes the boundary of the plate's middle surface. The bending moment per unit length $M$ [6] reads

$$
M=-D\left(\frac{\partial^{2} w}{\partial \boldsymbol{n}^{2}}+v \frac{\partial^{2} w}{\partial \tau^{2}}\right)
$$

and quantities $\boldsymbol{n}$ and $\boldsymbol{\tau}$ are the outer normal and tangent directions to $\partial S$, respectively, as shown in Fig. 4. Static and dynamic deflections under uniformly distributed pressure are targeted in this study; accordingly, due to straightforward considerations on the symmetry of the plate's geometry, on the external applied forces, and on the boundary conditions, it is legitimate to expand the displacements in series of sine functions $[6,10]$

$$
\begin{gathered}
w(x, y, t)=\sum_{n, m \in N} w_{2 n+1,2 m+1}(t) \sin \frac{(2 n+1) \pi x}{a} \sin \frac{(2 m+1) \pi y}{b} ; \\
u(x, y, t)=\sum_{n, m \in N} u_{2 n, 2 m+1}(t) \sin \frac{2 n \pi x}{a} \sin \frac{(2 m+1) \pi y}{b} \\
v(x, y, t)=\sum_{n, m \in N} v_{2 n+1,2 m}(t) \sin \frac{(2 n+1) \pi x}{a} \sin \frac{2 m \pi y}{b} .
\end{gathered}
$$

The problem is made non-dimensional by introducing a non-dimensional time $\tau=\Omega_{1} t$, where $\Omega_{1}$ is the circular frequency of the first natural mode of the deformed (pressure loaded) plate. Also, a change of notation has been introduced; the twosubscript generalized coordinates (time functions) $w_{2 n+1,2 m+1}, u_{2 n, 2 m+1}, v_{2 n+1,2 m}$ are divided by the plate thickness $h$ and replaced by the single-subscript generalized coordinates $q_{i}$ with corresponding increasing numbering.

\subsection{Static analysis}

First, the problem of static plate bending under uniform pressure is explored. As illustrated in Fig. 5, a quick convergence analysis shows that a 12 degree-of-freedom (dof) model stands as a convincing compromise between prediction capabilities and computational cost for the force-deflection curve of the Neo-Hookean plate.

The generalized coordinates plugged into the expansion of the displacements are given in Table 3. Responses are calculated with the LMM, starting from the configuration with $w_{1,1}=20 h$, captured by the less sophisticated model involving geometrical nonlinearity only. Accordingly, the curves start at central deformation of $20 \mathrm{~h}$ in Fig. 5. The maximal discrepancy between the 12 dof and 27 dof models in the deflection range $[0,100 h]$ is 2.3 percent. Similar results are found for other material models.

Force-deflection relationships for different materials with 12 dof are depicted in Fig. 6 . The displacement sensitivity to physical nonlinearities is highlighted by plotting the solution for linear elastic material and geometric nonlinearities only. Neo-Hookean and Mooney-Rivlin results almost coincide, while the Ogden curve slightly deviates for large deflections. Also, it is worthy to note that the LMM results agree very well with the exact solution.

Material nonlinearity for deflections smaller than $20 \mathrm{~h}$ can be neglected as shown in Fig. 6. For deflections up to $20 \mathrm{~h}$ the model with only geometrical nonlinearity is a very good approximation. As a consequence, the configuration computed with the linear elastic material is used as an initial guess for LMM numerical iterations for hyperelastic materials [1].

An additional validation of the results is carried out. The static deflection of the hyperelastic plate is also explored with the commercial finite element solution ANSYS [11] for Neo-Hookean and Ogden materials. The central deflection with respect to the applied external force is displayed in Fig. 7: FEA and LMM predictions are in good agreement.

The contribution of the in-plane nonlinearities in expressions (1) is now estimated. As an illustrative example, the pressure-deflection curves for the Neo-Hookean law with and without in-plane nonlinearities are shown in Fig. 8. The maximal difference is 2 percent. Other materials feature discrepancies of the same order. Despite of their relatively small influence, in-plane nonlinearities are incorporated in all numerical investigations, except in Fig. 8. 


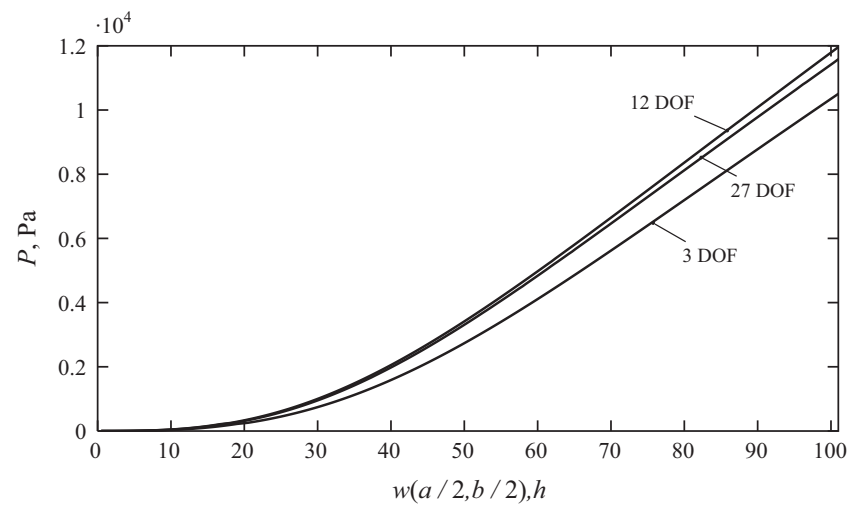

Fig. 5. Neo-Hookean plate pressure-deflection response. Deflection measured at the center of the plate and normalized with respect to the plate thickness $h$.

Table 3

Generalized coordinates utilized in the models.

\begin{tabular}{ll}
\hline Size & Participating eigenmodes \\
\hline 3 dof & $w_{1,1}, u_{2,1}, v_{1,2}$ \\
12 dof & $w_{i, j}, i, j=1,3 ; u_{i, j}, v_{j, i}, i=2,4 ; j=1,3$. \\
27 dof & $w_{i, j}, i, j=1,3,5 ; u_{i, j}, v_{j, i}, i=2,4,6 ; j=1,3,5$. \\
\hline
\end{tabular}

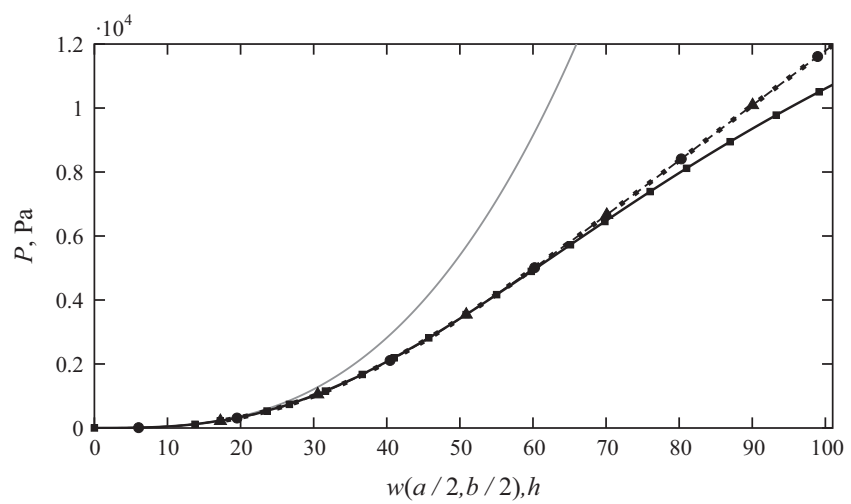

Fig. 6. Pressure-displacement response. LMM solution is shown in solid lines while exact results are given by symbols: $\bullet$, Neo-Hookean, $\bullet$, Mooney-Rivlin, -, Ogden models. Gray line: linear elastic material.

\subsection{Nonlinear vibration analysis}

At small strains, the effect of physical nonlinearities is negligible (see Fig. 6), and the vibrations of the initially flat plate can be explored by only retaining geometrical nonlinearities in the model [6]. Here, we focus on the investigation of a more challenging configuration where both nonlinearities are participating, i.e. the vibrations around a pre-loaded state. The deflection with principal generalized coordinate $w_{1,1}=80 h$ is chosen as an initial deformed configuration. Comparison with the exact static solution shows that the local model around this deformed configuration is accurate for deflections up to $10 \mathrm{~h}$, which therefore stands as an upper limit in the dynamic analysis. From previous analysis (see Fig. 5), it follows that the 12-dof model is sufficiently accurate and can be selected in order to reduce computation costs.

The harmonic balance method [12] is implemented to find the sought periodic solutions to system (10) through a Fourier expansion of the generalized coordinates in time

$$
q_{i}=A_{i 0}+\sum_{j=1}^{N_{h}} A_{i j} \cos (j \Omega \tau), \quad i=1, . . N
$$

where $\Omega$ is the non-dimensional frequency, normalized with respect to $\Omega_{1}$. Expression (20) considers cosine terms only since the external force and damping in (10) are ignored in the free vibration analysis. Coefficients $A_{i j}$ are determined from 


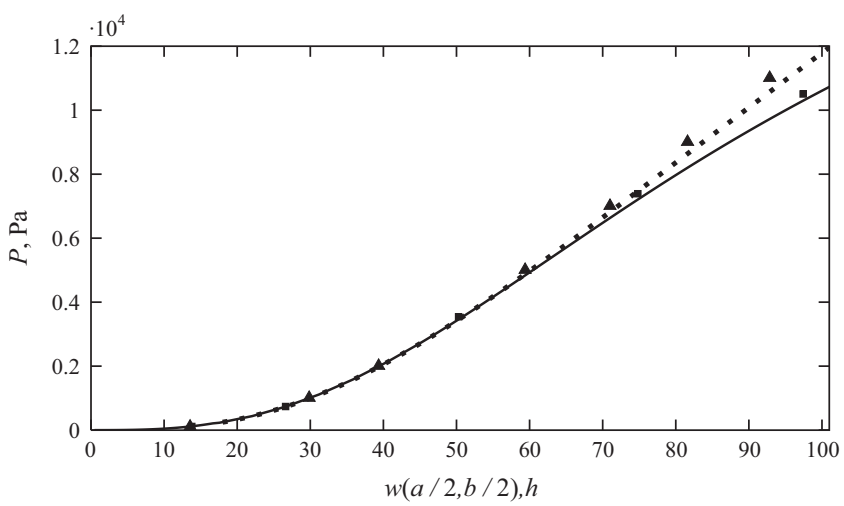

Fig. 7. Comparison between ANSYS and the exact solution. Dotted line corresponds to exact Neo-Hookean solution; solid line corresponds to exact Ogden solution; 4 , ANSYS with Neo-Hookean model; - ANSYS with Ogden model.

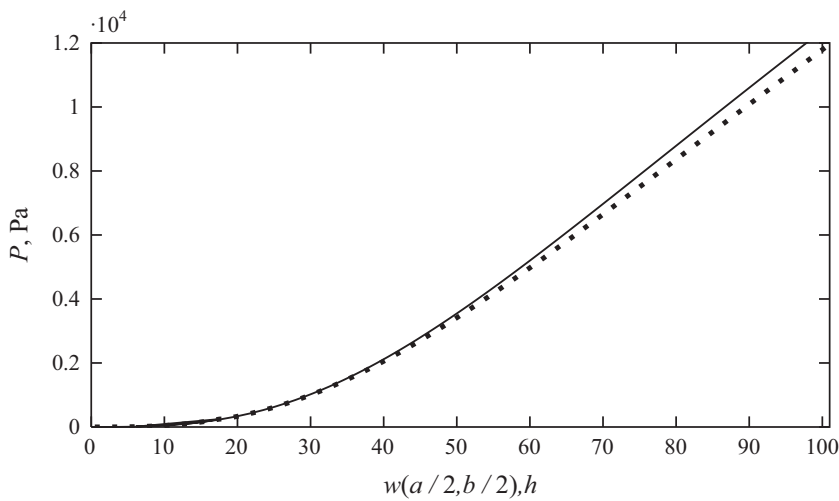

Fig. 8. Pressure-deflection response for Neo-Hookean material: with (dotted line) and without (solid line) in-plane nonlinearities.

Table 4

First eigenfrequency of the deformed rubber plate with $w_{1,1}=80 \mathrm{~h}$

\begin{tabular}{ll}
\hline Model & Natural frequency (rad/s) \\
\hline Neo-Hookean & 951.81 \\
Mooney-Rivlin & 951.78 \\
Ogden & 884.62 \\
\hline
\end{tabular}

the system of nonlinear algebraic equations resulting from balancing the coefficients associated to the same harmonics in Eq. (10) where expression (20) has been previously substituted. A convergence analysis shows that $N_{h}=4$ in (20) stands as an acceptable approximation of the solution.

The first eigenfrequencies of the deformed plate are given in Table 4 for an initial deflection $w_{1,1}=80 \mathrm{~h}$.

Fig. 9 displays the backbone curves of the plate's free vibrations, with frequencies close to the first eigenfrequency of the pre-loaded plate, with respect to non-dimensional frequencies, normalized with respect to the natural frequency $\Omega_{1}$ of the corresponding deflected plate.

Comparison to the exact static solution shows that Neo-Hookean and Mooney-Rivlin local models are accurate for deflections up to $10 h$, while Ogden model can only be considered for deflections not higher than $8 h$ only.

Again, Neo-Hookean and Mooney-Rivlin models yield identical results. This is true for both the eigenfrequency of the deformed plate and the backbone curve. Ogden backbone curve exhibits slightly weaker nonlinearity.

For all materials, the nonlinear nature of the deformed plate, as opposed to its flat counterpart [6], is very weak: the frequency of large-amplitude vibrations is almost identical to the natural frequency. In order to show the sensitivity of the backbone curves to the initial deflection, they are constructed around various initial configurations in Fig. 10. The NeoHookean material is selected since other materials exhibit similar features. Two effects are observed: the first one consists of an increased range of vibratory amplitudes where the backbone curve displays a softening behavior. This effect is wellknown and might be attributed to the contribution of the quadratic terms in (10) with respect to the initial deflection amplitude [6]. The second effect is the attenuation of the nonlinear nature of the model with an increased initial deflection. 


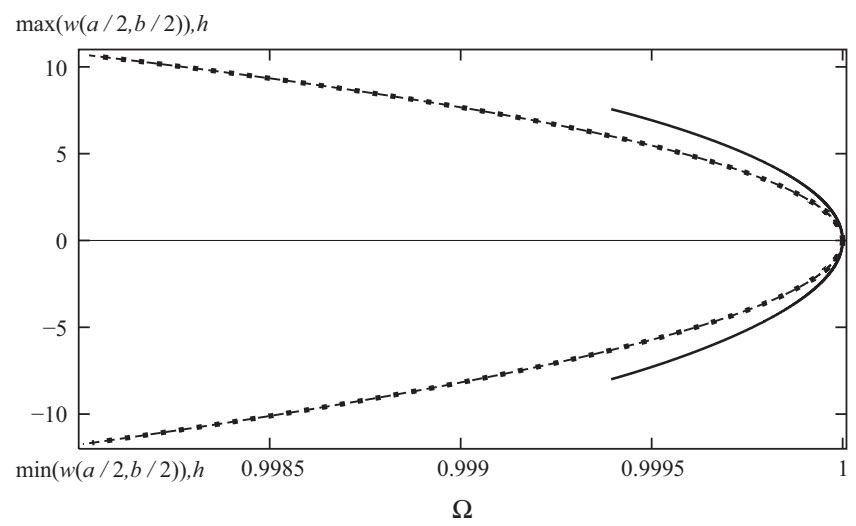

Fig. 9. Dimensionless backbone curves for free vibrations around the deformed configuration with $w_{1,1}=80 \mathrm{~h}$ for different models for rubber. Dotted line, Neo-Hookean law; dashed line, Mooney-Rivlin law; solid line, Ogden law.

(a)

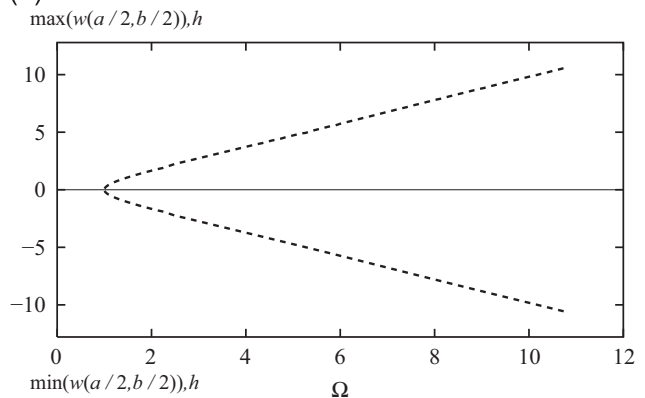

(c)

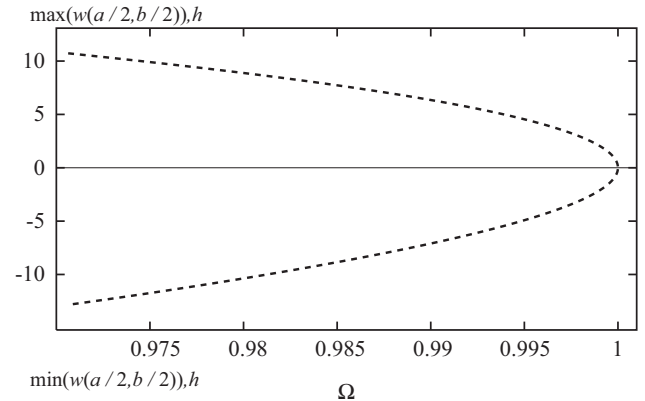

(b)

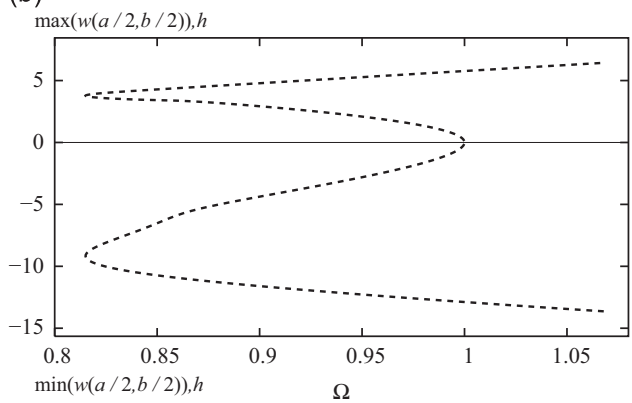

(d)

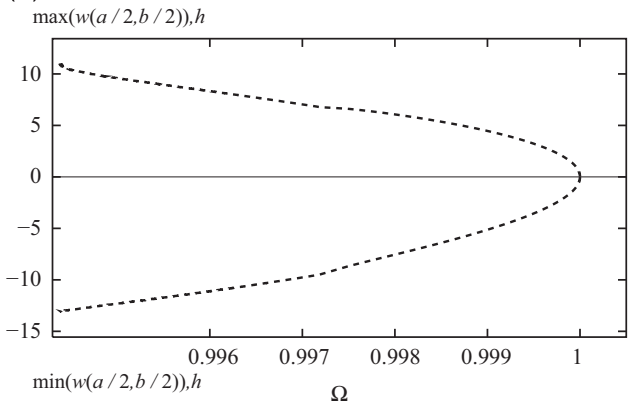

Fig. 10. Plate's free vibration backbone curves in the vicinity of various initially deformed configurations; Neo-Hookean model. (a) $w_{1,1}=0$ (flat plate); (b) $w_{1,1}=5 h$; (c) $w_{1,1}=30 h$; (d) $w_{1,1}=55 h$.

A similar behavior has been previously reported for bended plates [1] and stretched membranes [7] and is related to the large in-plane stretching associated to the initial deflection of the loaded plate.

The forced vibrations are also explored with the AUTO package [13]. External forcing derives from a time-dependent periodic pressure having an harmonic component $P_{d}=4.53 \mathrm{~Pa}$ and a constant mean-value corresponding to the deformed configuration, $w_{1,1}=80 h$, around which the local model is built. A modal damping ratio $\zeta_{n}=\zeta=0.001$ is adopted. The frequency response for the principal bending coordinate $w_{1,1}$ is shown in Fig. 11 . The vibratory response around a highly deformed configuration is almost identical to the linear response, in contrast to the response around a moderately deflected plate for which a significant softening behavior turning to hardening for vibration amplitude around $5 h$ is found as shown in Fig. 12.

\section{Static and dynamic plate bending for biological-type material}

\subsection{Hyperelastic models parameters}

The experimental data for uniaxial tension available in [14] are exploited. The present study is intended to model only one key feature of biological materials, i.e., a sharp increase in stiffness after a given strain threshold is reached. To this end, 


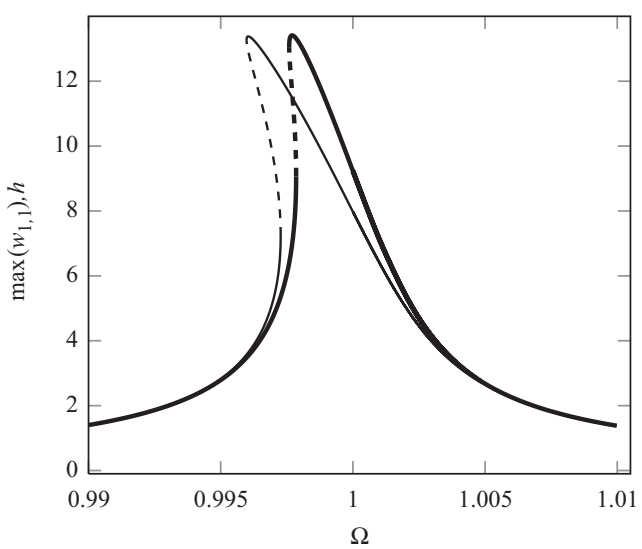

Fig. 11. Forced frequency response around deformed configuration with $w_{1,1}=80 h ; P_{d}=4.53 \mathrm{~Pa}, \zeta=0.001$. Generalized coordinate $w_{1,1}$. Continuous line, stable solution; dashed line, unstable solution. Thin line corresponds to Neo-Hookean law, thick line - to Ogden's law.

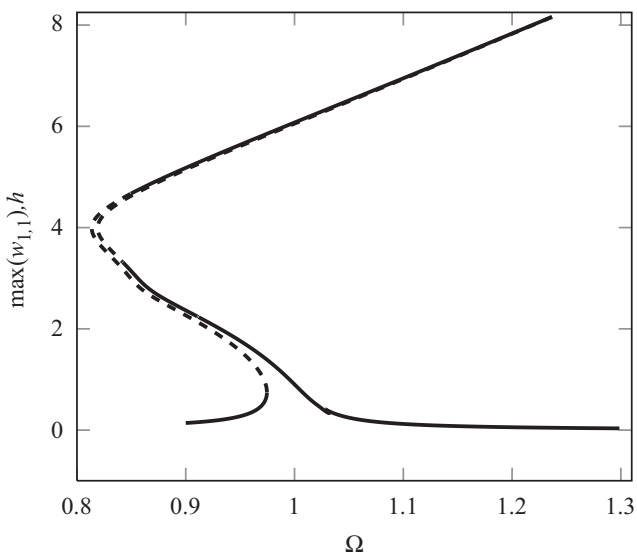

Fig. 12. Forced frequency response around deformed configuration with $w_{1,1}=5 h ; P_{d}=0.063 \mathrm{~Pa}, \zeta=0.001$. Generalized coordinate $w_{1,1}$. Continuous line, stable solution; dashed line, unstable solution.

\section{Table 5}

Biomaterial parameters of hyperelastic laws.

\begin{tabular}{ll}
\hline Neo-Hookean & $E=59,383.2 \mathrm{~Pa}$ \\
Mooney-Rivlin & $\mu_{1}=25,829.8 \mathrm{~Pa}, \mu_{2}=-6035.4 \mathrm{~Pa}$ \\
Ogden & $\mu_{1}=466,515,161.8 \mathrm{~Pa}, \quad \mu_{2}=2.49 \times 10^{-9} \mathrm{~Pa}$, \\
& $\mu_{3}=-1,115,907,998.2 \quad \mathrm{~Pa}, \mu_{4}=649,412,722.6 \quad \mathrm{~Pa}$, \\
& $\alpha_{1}=7.385, \quad \alpha_{2}=84.402, \quad \alpha_{3}=7.365, \quad \alpha_{4}=7.35$ \\
& \\
\hline
\end{tabular}

the experimental data points that corresponding to the tunica adventitia of a human aorta in [14] is selected. The procedure described in Appendix A is implemented with the difference that only the uniaxial test data are available. Corresponding material parameters are listed in Table 5.

The associated stress-strain relationships are shown in Figs. 13-15. The difference between Neo-Hookean and MooneyRivlin laws is clearly distinguishable for this type of material. However, both laws are in agreement with the experimental points for strains less than 8 percent and both are unable to reproduce the increase in stiffness, as opposed to Ogden law.

\subsection{Static analysis}

The problem detailed in Section 6.2 is solved for the hyperelastic materials listed in Table 5. Attention is paid to the static bending of the plate (Fig. 4) with the help of the 12 dof model (Table 3). The exact pressure-deflection response is depicted in Fig. 16.

Neo-Hookean and Mooney-Rivlin results are almost identical, while Ogden model starts to depart from these curves for amplitudes about 80h, as displayed in Fig. 16, where is becomes much stiffer. Strains at the point where the Ogden and the 


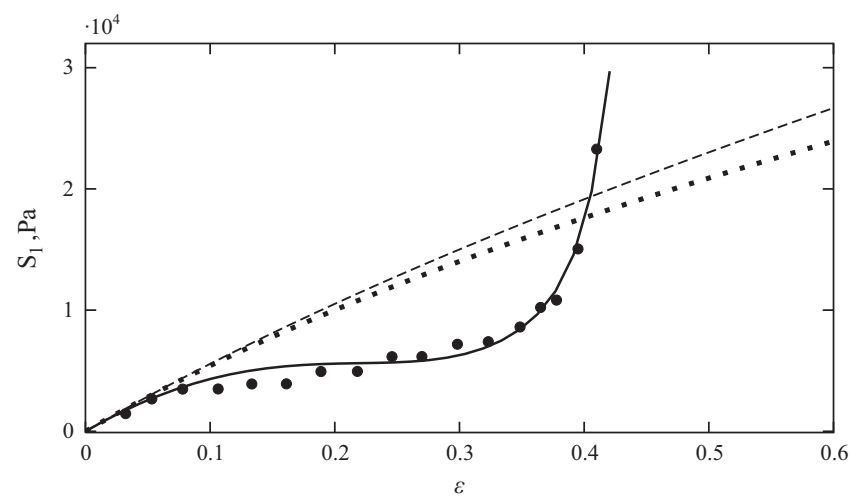

Fig. 13. Strain-stress curves and experimental points for uniaxial tension of biomaterial. Dotted line, Neo-Hookean law; dashed line, Mooney-Rivlin law; continuous line, Ogden law; •, experimental point.

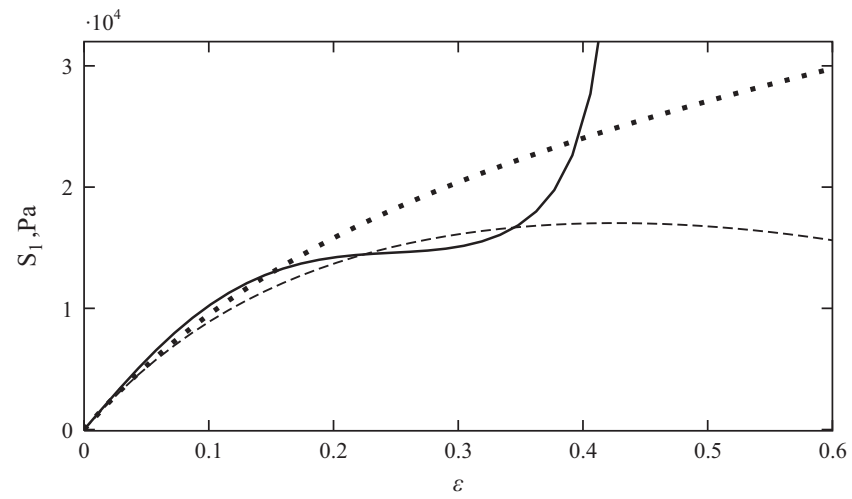

Fig. 14. Strain-stress curves for equibiaxial tension of biomaterial. Dotted line, Neo-Hookean law; dashed line, Mooney-Rivlin law; continuous line, Ogden law.

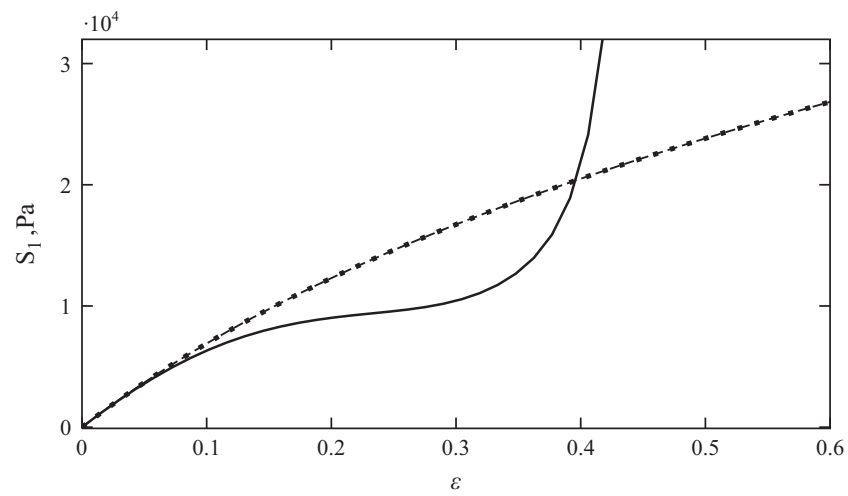

Fig. 15. Strain-stress curves for pure shear of biomaterial. Dotted line, Neo-Hookean law; dashed line, Mooney-Rivlin law; continuous line, Ogden law.

Neo-Hookean curves cross each other in Fig. 16 are also analyzed through the strain intensity, given by [15]

$$
\varepsilon_{\mathrm{INT}}=\sqrt{\frac{2}{3}} \sqrt{\left(\varepsilon_{1}-\varepsilon_{2}\right)^{2}+\left(\varepsilon_{1}-\varepsilon_{3}\right)^{2}+\left(\varepsilon_{2}-\varepsilon_{3}\right)^{2}+\frac{3}{2} \varepsilon_{12}}
$$

At the crossing point (Fig. 16) in the center of the plate $\varepsilon_{\mathrm{INT}}=42.5$ percent, which is close to the values of the crossing points in the stress-strain curves, which are 40 percent for uniaxial test and 39.6 percent for equibiaxial test (see Figs. 13 and 14). 


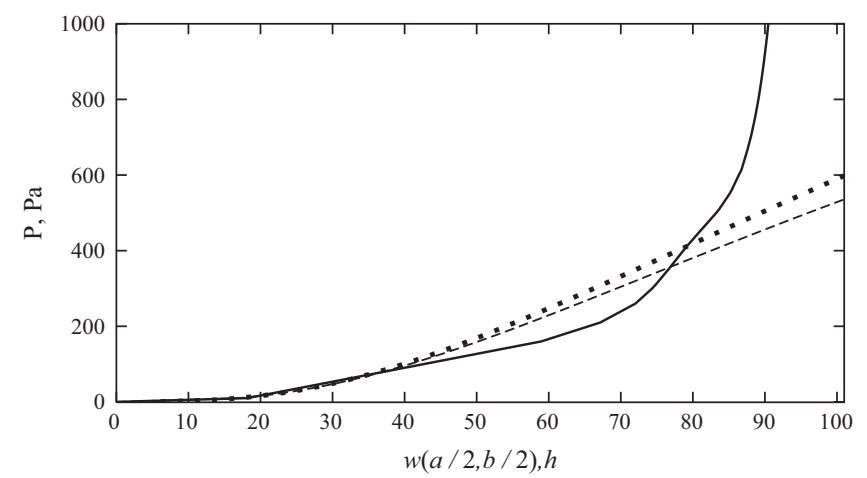

Fig. 16. Pressure load versus central deflection for various hyperelastic biomaterial models, 12 dof. Dotted line, Neo-Hookean law; dashed line, MooneyRivlin law; continuous line, Ogden law.

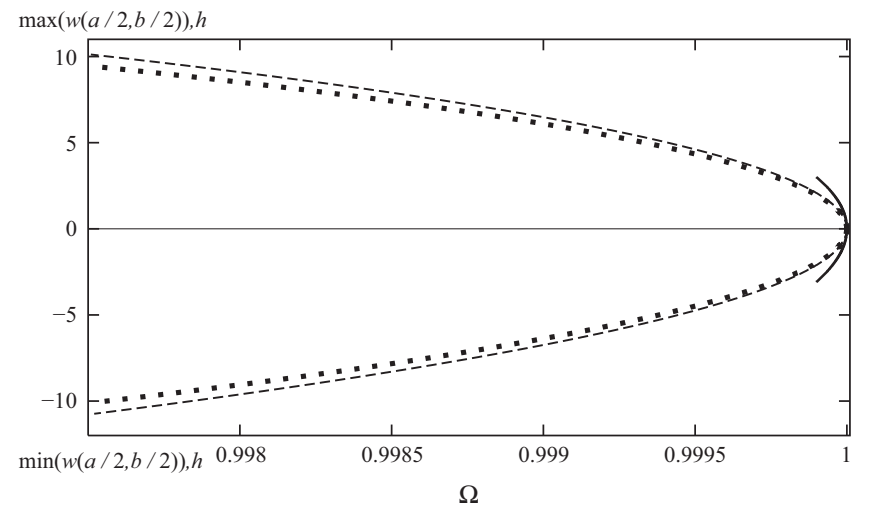

Fig. 17. Biomaterial square plate free vibration backbone curves around the deformed configuration with $w_{1,1}=70 h$. Dotted line, Neo-Hookean law; dashed line, Mooney-Rivlin law; solid line, Ogden law.

Table 6

First eigenfrequency of the deformed biomaterial plate with $w_{1,1}=70 h$.

\begin{tabular}{ll}
\hline Model & Natural frequency (rad/s) \\
\hline Neo-Hookean & 183 \\
Mooney-Rivlin & 177.88 \\
Ogden & 181.96 \\
\hline
\end{tabular}

\subsection{Vibration analysis}

We investigate free nonlinear vibrations around the deformed response with principal generalized coordinate $w_{1,1}=70 h$. The comparison with the exact static solution shows that Neo-Hookean and Mooney-Rivlin local models are accurate for deflections up to $10 \mathrm{~h}$ whereas Ogden model is limited to deflections not larger $3 \mathrm{~h}$ only. The corresponding backbone curves are obtained through the harmonic balance method (20) and are depicted in Fig. 17 for vibrations around the first eigenfrequency of the pre-loaded plate. The first eigenfrequency of the deformed plate is listed in Table 6 for the three hyperelastic laws. The mass density is $\rho=1380 \mathrm{~kg} / \mathrm{m}^{3}$, which is the density of Polyethylene terephthalate, frequently used for artificial arteries [16]. We notice that Neo-Hookean and Mooney-Rivlin results again are in good agreement for all studied range of deflections. The Ogden's plate eigenfrequencies are close to the predicted by these two models up to central deflection of $80 \mathrm{~h}$ and afterwards they are essentially higher.

Similarly to the example of the rubber plate, the behavior of a deformed plate made of biomaterial is weakly nonlinear for all materials and the nonlinearity is of softening type. However, certain features differ. Although static results for NeoHookean and Mooney-Rivlin models are almost identical, the MooneyRivlin backbone curve shows a softer behavior. Still, the Ogden curve is the softest one and is presented for vibration amplitude up to $3 h$ in Fig. 17. 


\section{Conclusions}

Vibration of plates made of rubber and biological materials are explored with a dedicated local models method. Local models allow for the analysis of static bending as well as physically and geometrically large-amplitude nonlinear vibrations of an initially distorted plate. Static results are validated through a systematic comparison with available exact solutions as well as against commercial FE software results. It is found that the local model method provides accurate predictions for a wide range of deflections. However, the sharp increase in stiffness peculiar to biological materials limits the range of achievable vibrations amplitudes.

In most cases, it is found that Mooney-Rivlin and Neo-Hookean materials exhibit similar static and vibratory behaviors. Corresponding constitutive laws properly capture the behavior of the actual material at moderate strains (30 percent for rubber and 8 percent for biomaterial). The best approximation is provided by Ogden model. The latter correctly reproduces the behavior at high strains, including the well-known sharp increase in stiffness. However, Ogden model has significant drawbacks. Due to the complicated form of the strain energy density and its formulation in terms of principal strains (unlike Neo-Hookean and Mooney-Rivlin models which allow for a formulation in terms of strain invariants), it is computationally much more expensive. It is also shown that the pre-loaded plate exhibits very weak dynamic nonlinearity, i.e. the frequencies of the oscillations around the deformed configuration are almost identical to the associated eigenfrequencies.

The sensitivity of the free vibration backbone curves to the initial deflection is also discussed. It is shown that the higher the initial static deflection, the higher the level of amplitudes at which the backbone curve displays a softening behavior. The nonlinear nature of the system of interest (the frequency shift between low- and large-amplitude vibrations) weakens with an increased initial deflection.

\section{Acknowledgments}

The authors acknowledge the financial support of NSERC Discovery Grant, Canada Research Chair, Canada Foundation for Innovation (LOF) programs of Canada and the PSR-SIIRI program of Québec.

\section{Appendix A. Hyperelastic material parameter identification}

The following nominal stress expressions for Neo-Hookean law are used in order to fit the experimental data (uniaxial, equibiaxial tensions and pure shear, respectively) [7,8]:

$$
\begin{aligned}
& S_{1}=\frac{E}{3}\left((\varepsilon+1)-(\varepsilon+1)^{-2}\right), \\
& S_{1}=\frac{E}{3}\left((\varepsilon+1)-(\varepsilon+1)^{-5}\right), \\
& S_{1}=\frac{E}{3}\left((\varepsilon+1)-(\varepsilon+1)^{-3}\right) .
\end{aligned}
$$

For Mooney-Rivlin law, these expressions become [7,8]

$$
\begin{gathered}
S_{1}=\mu_{1}\left((\varepsilon+1)-(\varepsilon+1)^{-2}\right)+\mu_{2}\left(1-(\varepsilon+1)^{-3}\right), \\
S_{1}=\mu_{1}\left((\varepsilon+1)-(\varepsilon+1)^{-5}\right)+\mu_{2}\left((\varepsilon+1)^{3}-(\varepsilon+1)^{-3}\right), \\
S_{1}=\left(\mu_{1}+\mu_{2}\right)\left((\varepsilon+1)-(\varepsilon+1)^{-3}\right) .
\end{gathered}
$$

While for Ogden law, they are [7]

$$
\begin{gathered}
S_{1}=\sum_{i=1}^{N_{T}} \mu_{i}\left((\varepsilon+1)^{\alpha_{i}-1}+(\varepsilon+1)^{\left(-\alpha_{i} / 2\right)-1}\right), \\
S_{1}=\sum_{i=1}^{N_{T}} \mu_{i}\left((\varepsilon+1)^{\alpha_{i}-1}+(\varepsilon+1)^{-2 \alpha_{i}-1}\right), \\
S_{1}=\sum_{i=1}^{N_{T}} \mu_{i}\left((\varepsilon+1)^{\alpha_{i}-1}+(\varepsilon+1)^{-\alpha_{i}-1}\right) .
\end{gathered}
$$

The procedure for the determination of the material parameters is as follows. First, Young's modulus is determined using the expressions for Neo-Hookean law. For this purpose, the material is assumed to be Neo-Hookean and E is determined through a least squares technique by using only one point (corresponding to the smallest stress) for each stress-strain test. For the sake of consistency, Young's modulus is assumed to be identical for all the hyperelastic laws. This yields $3\left(\mu_{1}+\mu_{2}\right)=E$ for Mooney-Rivlin and $3 / 2 \sum_{i=1}^{N_{T}} \mu_{i} \alpha_{i}=E$ for the Ogden law [7,8]. Once $E$ is known, the Neo-Hookean strain energy density is 
completely defined. The Mooney-Rivlin law contains one unknown parameter and the Ogden law involves $2 N_{T}-1$ unknowns. These parameters are determined by the least squares fitting of the expressions (A4)-(A9) to the experimental data.

\section{References}

[1] I. Breslavsky, M. Amabili, M. Legrand, Physically and geometrically non-linear vibrations of thin rectangular plates, International Journal of Non-linear Mechanics 58 (2014) 30-40.

[2] D.R. Einstein, P. Reinhall, M. Nicosia, R.P. Cochran, K. Kunzelman, Dynamic finite element implementation of nonlinear, anisotropic hyperelastic biological membranes, Computer Methods in Biomechanics and Biomedical Engineering 6 (2003) 33-44.

[3] E. Verron, G. Marckmann, B. Peseux, Dynamic inflation of non-linear elastic and viscoelastic rubber like membranes, International Journal for Numerical Methods in Engineering 50 (2001) 1233-1251.

[4] P.B. Goncalves, R.M. Soares, D. Pamplona, Nonlinear vibrations of a radially stretched circular hyperelastic membrane, Journal of Sound and Vibration 327 (2009) 231-248.

[5] R.M. Soares, P.B. Goncalves, Nonlinear vibrations and instabilities of a stretched hyperelastic annular membrane, International Journal of Solids and Structures 49 (2012) 514-526.

[6] M. Amabili, Nonlinear Vibrations and Stability of Shells and Plates, Cambridge University Press, New York, 2008.

[7] R. Ogden, Non-Linear Elastic Deformations, Dover Publications, New York, 1997.

[8] A. Bower, Applied Mechanics of Solids, CRC Press. Taylor and Francis Group, Boca Raton, 2010.

[9] L.R.G. Treloar, Stress-strain data for vulcanised rubber under various types of deformation, Transactions of the Faraday Society 40 (1944) 59-70.

[10] I.D. Breslavsky, K.V. Avramov, Two modes nonresonant interaction for rectangularplate with geometrical nonlinearity, Nonlinear Dynamics 69 (2012) $285-294$.

[11] S. Moaveni, Finite Element Analysis Theory and Application with ANSYS, 3rd ed. Prentice Hall, Upper Saddle River, New Jersey, 2007.

[12] T.S. Parker, L.O. Chua, Practical Numerical Algorithms for Chaotic Systems, Springer-Verlag, New York, 1989.

[13] E.J. Doedel, A.R. Champneys, T.F. Fairgrieve, Y.A. Kuznetsov, B. Sandstede, X. Wang, AUTO 97: Continuation and Bifurcation Software for Ordinary Differential Equations (with HomCont), Concordia University, Montreal, 1998.

[14] G.A. Holzapfel, Determination of material models for arterial walls from uniaxial extension tests and histological structure, Journal of Theoretical Biology 238 (2006) 290-302.

[15] L. Kachanov, Fundamentals of Theory of Plasticity, Courier Dover Publications, Mineola, 2004.

[16] J. Chlupáč, E. Filová, L. Bačáková, Blood vessel replacement: 50 years of development and tissue engineering paradigms in vascular surgery, Physiological Research 58 (2009) S119-S139. 